\title{
TOPOLOGICAL CENTERS OF CERTAIN DUAL ALGEBRAS
}

\author{
ANTHONY TO-MING LAU AND ALI ÜLGER
}

\begin{abstract}
Let $A$ be a Banach algebra with a bounded approximate identity. Let $Z_{1}$ and $\widetilde{Z}_{2}$ be, respectively, the topological centers of the algebras $A^{* *}$ and $\left(A^{*} A\right)^{*}$. In this paper, for weakly sequentially complete Banach algebras, in particular for the group and Fourier algebras $L^{1}(G)$ and $A(G)$, we study the sets $Z_{1}, \widetilde{Z}_{2}$, the relations between them and with several other subspaces of $A^{* *}$ or $A^{*}$.
\end{abstract}

\section{INTRODUCTION}

Let $A$ be a Banach algebra with a bounded approximate identity (= BAI). Terminologies and notations not explained in this section will be explained or referenced in the next section. For $f$ in $A^{*}$ and $a$ in $A$ we denote by $f \cdot a$ the element of $A^{*}$ defined by $\langle f \cdot a, x\rangle=\langle f, a x\rangle$. By $A^{*} A$ we denote the subspace of $A^{*}$ consisting of the functionals of the form $f \cdot a$, for all $f$ in $A^{*}$ and $a$ in $A$. This is known to be a norm closed linear subspace of $A^{*}[17 ; 32.22]$. As is well-known [1], the second dual $A^{* *}$ of $A$ endowed with the either Arens multiplications is a Banach algebra. Unless otherwise stated, we shall work with the first Arens multiplication, the construction of which is given in the next section. The dual of the space $A^{*} A$ equipped with the multiplication induced by that of $A^{* *}$ is also a Banach algebra. The topological centers of $A^{* *}$ and $\left(A^{*} A\right)^{*}$ are defined, respectively, by

$$
\begin{array}{ll}
Z_{1}=\left\{m \in A^{* *}: \quad \begin{array}{l}
\text { The mapping } n \mapsto m \cdot n \text { is } \\
\text { weak } \left.{ }^{*} \text {-weak* continuous on } A^{* *}\right\} .
\end{array}\right. \\
\widetilde{Z}_{1}=\left\{\mu \in\left(A^{*} A\right)^{*}: \begin{array}{l}
\text { The mapping } n \mapsto \mu \cdot n \text { is } \\
\text { weak }
\end{array}\right. \\
\end{array}
$$

It happens that the sets $Z_{1}$ and $\widetilde{Z}_{1}$ are very closely connected to each other and also to the algebra of all right multipliers of $A$.

Our purpose in this paper can be summarized as follows. a) To find out when the equality $A^{*}=A^{*} A$ or $A^{*}=A A^{*}$ holds; in which case $Z_{1}$ and $\widetilde{Z}_{1}$ coincide.

Received by the editors September 4, 1994 and, in revised form, March 27, 1995.

1991 Mathematics Subject Classification. Primary 43A20; Secondary 46H05.

Key words and phrases. Topological center, multiplier algebra, group algebra and Fourier algebra.

The research of the first author is supported by an NSERC grant.

The research of the second author is supported by TUBA. 
b) To study the relations of the algebra $\widetilde{Z}_{1}$ with $Z_{1}$ and also with the algebra $\operatorname{RM}(A)$ of the right multipliers of $A$. c) To find out when the equality $A^{*} A=\operatorname{wap}(A)$ is equivalent to $A$ being an ideal in its second dual. d) To characterize the elements of $A$ or $Z_{1}$ among those of $A^{* *}$; and e) To exhibit the exact role played by each ingredient that plays some role in the study of the sets $Z_{1}$ and $\widetilde{Z}_{1}$. Throughout the paper, particular attention will be given to the case when $A$ is the group algebra $L^{1}(G)$ or the Fourier algebra $A(G)$ of a locally compact group $G$; and also to the case when $A$ or $A^{*}$ is weakly sequentially complete.

In recent years, the topological center problem for the algebras $A^{* *}$ and $\left(A^{*} A\right)^{*}$ has attracted some attention. The most studied cases seem to be the group algebra $L^{1}(G)$, the Fourier algebra $A(G)$ and the corresponding algebras $\left(A^{*} A\right)^{*}=$ $\operatorname{LUC}(G)^{*}$ and $\left(A^{*} A\right)^{*}=\operatorname{UCB}(\widehat{G})^{*}$. In $[29]$ Zappa proved that, for $A=L^{1}(\mathbb{R}), \widetilde{Z}$ $=M(\mathbb{R})$, the measure algebra of $\mathbb{R}$. In [14] Grosser and Losert extended this result to all locally compact abelian groups and in [20] Lau extended it to all locally compact groups. In [25] Parsons proved that, for certain abelian discrete groups $G$, for the algebra $A=\ell^{1}(G), Z_{1}=A$. In [18] Isik, Pym and Ülger showed that, for any compact group $G$, the topological center of $L^{1}(G)^{* *}$ is $L^{1}(G)$. This result has been extended to all locally compact groups by Lau and Losert in [21]. The topological center problem for the Fourier algebra $A(G)$ has been studied by Cecchini and Zappa in [3] and Lau and Losert in [22]. Their results show that for a large class of locally compact groups $G$, including the motion group, the " $a x+b$ "-group and the Heisenberg group, the topological center of the algebra $A(G)^{* *}$ is $A(G)$ and that of $\operatorname{UCB}(\widehat{G})^{*}$ is $B(G)$, the Fourier-Stieltjes algebra of $G$. The cases of the Volterra algebra $V$ and the weighted group algebra $L^{1}\left(\mathbb{R}^{+}, w\right)$ have been studied by Ghahramani and McClure in [8]. Their results show that for these algebras also $Z_{1}$ is again the algebra itself.

In this paper we study the topological center problem for the algebras $A^{* *}$ and $\left(A^{*} A\right)^{*}$ from an abstract point of view, paying special attention to weakly sequentially complete Banach algebras and, in particular, to the group algebra $L^{1}(G)$ and the Fourier algebra $A(G)$.

This paper is organized as follows. In Section 2 we study the question when $A^{*}$ factors on the left or right. That is, the question when we have $A^{*}=A^{*} A$ or $A^{*}=A A^{*}$. As we shall see, the equality $A^{*}=A^{*} A$ does not imply, nor is implied by, the equality $A^{*}=A A^{*}$ and that the equalities $A^{*}=A^{*} A=A A^{*}$ are possible even if $A$ is not unital or Arens regular. We shall also see that if $A^{*}$ factors on the one side but not on the other side, then the topological centers $Z_{1}$ and $Z_{2}$ of $A^{* *}$ with respect to the first and second Arens multiplications are different. In Section 3 we study the relationship of $Z_{1}$ with $\widetilde{Z}_{1}$ and the role of weak sequential completeness in the study of the topological center problem. We also provide an answer to the question when the equality wap $(A)=A^{*} A$ is equivalent to the fact that $A$ is a right ideal in its second dual. In Section 4 we study the relationship of $\widetilde{Z}_{1}$ with the algebra $\mathrm{RM}(A)$ of right multipliers of $A$. Here we show that $\operatorname{RM}(A)$ is isometrically isomorphic to a subalgebra of $\widetilde{Z}_{1}$ if the BAI of $A$ is bounded by 1 . In particular, when $Z_{1}=A$ then $\widetilde{Z}_{1}=\mathrm{RM}(A)$. In Section 5 we try to characterize the elements of $Z_{1}$ (resp. $A$ ) among those of $A^{* *}$. As applications of the results obtained, we give two new proofs of the fact that the topological center of $L^{1}(G)^{* *}$ is $L^{1}(G)$ and also, for certain groups $G$, of the fact that the topological center of $A(G)^{* *}$ is $A(G)$. The paper ends with some remarks and open questions. 


\section{Notation and Preliminary Results}

In this section we have collected some notation and results we need in the subsequent sections. Our notation and terminology are quite standard. For any Banach space $X$, by $X^{*}$ we denote its normed dual. We always regard $X$ as naturally embedded into its second dual $X^{* *}$. For $x$ in $X$ and $f$ in $X^{*}$, indifferently, by $\langle x, f\rangle$ and $\langle f, x\rangle$ we denote the natural duality between $X$ and $X^{*}$. For any two Banach spaces $X$ and $Y$, by $X \stackrel{\vee}{\otimes} Y$ and $X \widehat{\otimes} Y$ we denote, respectively, the injective and projective tensors products of $X$ and $Y$ [6]. By $G$ we denote a locally compact group equipped with a left Haar measure. The spaces $C_{00}(G), C_{0}(G), L^{p}(G)(1 \leq p \leq \infty)$ and $M(G)$ are as in Hewitt and Ross [17]. The Fourier and the Fourier-Stieltjes algebras $A(G)$ and $B(G)$ of $G$ are as in Eymard [7]. For any Banach algebra $A$, for $a$ in $A$ and $f$ in $A^{*}$, we define the functionals $f \cdot a$ and $a \cdot f$ by $\langle f \cdot a, x\rangle=\langle f, a x\rangle$ and $\langle a \cdot f, x\rangle=\langle f, x a\rangle$. We define the subspaces $A^{*} A$ and $A^{*} A$ of $A^{*}$ as

$$
A^{*} A=\left\{f \cdot a: f \in A^{*} \text { and } a \in A\right\} \quad \text { and } \quad A A^{*}=\left\{a \cdot f: a \in A \text { and } f \in A^{*}\right\} .
$$

In the case where $A=L^{1}(G)$, we denote the spaces $A^{*} A$ and $A A^{*}$, respectively, by $\operatorname{LUC}(G)$ and $\operatorname{RUC}(G)$ as in [20]. In the case where $A=A(G)$, the space $A^{*} A$, which is the same as $A A^{*}$, is denoted by $\operatorname{UCB}(\widehat{G})$ as in [22]. A functional $f$ in $A^{*}$ is said to be weakly almost periodic if the set $\{f \cdot a: a \in A,\|a\| \leq 1\}$ is relatively weakly compact. We denote by wap $(A)$ the closed subspace of $A^{*}$ consisting of all the weakly almost periodic functionals in $A^{*}$. A bounded net $\left(e_{\alpha}\right)_{\alpha \in I}$ in $A$ is said to be a "bounded approximate identity" (= BAI) if for each $a$ in $A,\left\|e_{\alpha} a-a\right\| \rightarrow 0$ and $\left\|a e_{\alpha}-a\right\| \rightarrow 0$. We also need the constructions of the two Arens multiplications. The first Arens multiplication is defined as follows in three steps. For $a, b$ in $A, f$ in $A^{*}$ and $n, m$ in $A^{* *}$, the elements $f \cdot a, m \cdot f$ of $A^{*}$ and $m \cdot n$ of $A^{* *}$ are defined as follows:

$$
\begin{aligned}
\langle f \cdot a, b\rangle & =\langle f, a b\rangle, \\
\langle m \cdot f, a\rangle & =\langle m, f \cdot a\rangle, \\
\langle m \cdot n, f\rangle & =\langle m, n \cdot f\rangle .
\end{aligned}
$$

The basic properties of this multiplication are as follows. For $n$ fixed in $A^{* *}$, the mapping $m \mapsto m \cdot n$ is weak $^{*}$-weak ${ }^{*}$ continuous. For $m$ fixed in $A^{* *}$, the mapping $n \mapsto m \cdot n$ is in general not weak*-weak* continuous unless $m$ is in $A$. Whence the topological center of $A^{* *}$ with respect to this multiplication is defined as follows.

$$
\begin{aligned}
Z_{1}=\left\{m \in A^{* *}:\right. & \text { The mapping } n \mapsto m \cdot n \text { is weak }{ }^{*} \text {-weak }^{*} \\
& \text { continuous on } \left.A^{* *}\right\} .
\end{aligned}
$$

The second Arens multiplication is defined as follows. For $a, b$ in $A, f$ in $A^{*}$ and $m, n$ in $A^{* *}$, the elements $a \Delta f, f \Delta m$ of $A^{*}$ and $m \Delta n$ of $A^{* *}$ are defined by the equalities

$$
\begin{aligned}
\langle b, a \Delta f\rangle & =\langle b a, f\rangle, \\
\langle a, f \Delta m\rangle & =\langle a \Delta f, m\rangle, \\
\langle f, m \Delta n\rangle & =\langle f \Delta m, n\rangle .
\end{aligned}
$$


For $m$ fixed in $A^{* *}$, the mapping $n \mapsto m \Delta n$ is weak ${ }^{*}$-weak* continuous on $A^{* *}$. For $n$ fixed in $A^{* *}$, the mapping $m \mapsto m \Delta n$ is in general not weak ${ }^{*}$-weak ${ }^{*}$ continuous on $A^{* *}$ unless $n$ is in $A$. Whence the topological center of $A^{* *}$ with respect to this multiplication is defined as follows.

$$
\begin{aligned}
Z_{2}=\left\{n \in A^{* *}:\right. & \text { The mapping } m \mapsto m \Delta n \text { is weak }{ }^{*} \text {-weak } \\
& \text { continuous on } \left.A^{* *}\right\} .
\end{aligned}
$$

We also recall that, for $a$ in $A$ and $m$ in $A^{* *}, a \cdot m=a \Delta m$ and $m \cdot a=m \triangle a$. It is clear that $A \subseteq Z_{1} \cap Z_{2}$ and that $Z_{i} \quad(i=1,2)$ is a closed subalgebra of $A^{* *}$ endowed with the first (second) Arens multiplication. If, for each $n, m$ in $A^{* *}$, the equality $n \cdot m=n \triangle m$ holds the algebra $A$ is said to be Arens regular. In this case $Z_{1}=Z_{2}=A^{* *}$. This is also equivalent to saying that wap $(A)=A^{*}$, see $[26]$.

Finally we recall the definition of mixed units which play a fundamental role in this paper. An element $E$ of $A^{* *}$ is said to be a mixed unit if $E$ is a right unit for the first Arens multiplication and a left unit for the second Arens multiplication. That is, $E$ is a mixed unit if and only if, for each $m$ in $A^{* *}, m \cdot E=E \Delta m=m$. It is easy to see that an element $E$ of $A^{* *}$ is a mixed unit if and only if it is a weak* cluster point of some BAI $\left(e_{\alpha}\right)_{\alpha \in I}$ in $A$, see [2; p. 146].

Convention. Throughout the paper, $A^{* *}$ will denote the Banach algebra $A^{* *}$ equipped with the first Arens multiplication. When we consider $A^{* *}$ as a Banach algebra with respect to the second Arens multiplication it will be denoted as $\left(A^{* *}, \triangle\right)$.

\section{Factorization of the Dual of a Banach Algebra}

Let $A$ be a Banach algebra with a BAI. If the equality $A^{*} A=A^{*}$ (resp. $A A^{*}=$ $A^{*}$ ) holds then we say that $A^{*}$ factors on the left (resp. right) or is left (resp. right) essential. If both equalities $A^{*} A=A A^{*}=A^{*}$ hold then we say that $A^{*}$ factors. In this section, our aim is to present some necessary and sufficient conditions for $A^{*}$ to factor on the left or right and to study the relationship of this notion with the topological centers of $A^{* *}$.

Lemma 2.1. Let $A$ be a Banach algebra with a BAI $\left(e_{\alpha}\right)_{\alpha \in I}$. Then, $A^{*}$ factors on the left (resp. right) if and only if, for each $f$ in $A^{*}$, the net $\left(f \cdot e_{\alpha}\right)_{\alpha \in I}$ (resp. $\left.\left(e_{\alpha} \triangle f\right)_{\alpha \in I}\right)$ converges weakly to $f$.

Proof. We shall prove only the left version of the assertion, the proof of the right version is similar. Assume first that $A^{*}$ factors on the left and let $f$ be an element of $A^{*}$. Then $f$ is of the form $f=g \cdot a$ for some $g$ in $A^{*}$ and $a$ in $A$. Hence, since $\left\|a e_{\alpha}-a\right\| \rightarrow 0$, for any $m$ in $A^{* *}, \quad\left\langle f \cdot e_{\alpha}, m\right\rangle=\left\langle g, a e_{\alpha} m\right\rangle \rightarrow\langle g, a \cdot m\rangle=\langle f, m\rangle$, so that $f \cdot e_{\alpha} \rightarrow f$ weakly. Conversely, assume that, for each $f$ in $A^{*}, f \cdot e_{\alpha} \rightarrow f$ weakly. Then since $f \cdot e_{\alpha}$ is in $A^{*} A$ and since $A^{*} A$ is a closed subspace of $A^{*}$ [17; 32.22], $f$ is in $A^{*} A$. Hence $A^{*}=A^{*} A$ and $A^{*}$ factors on the left.

Proposition 2.2. Let $A$ be a Banach algebra with a BAI. Then the following assertions hold.

a) $A^{*}$ factors on the left if and only if the algebra $A^{* *}$ is unital.

b) $A^{*}$ factors on the right if and only if the algebra $\left(A^{* *}, \triangle\right)$ is unital. 
c) If $A^{*}$ factors then the unit elements of the algebras $A^{* *}$ and $\left(A^{* *}, \triangle\right)$ are the same.

Proof. a) Assume $A^{*}$ factors on the left. Since $A$ has a BAI, $A^{* *}$ has mixed units. Let $E$ be one of them. Then, for $m$ in $A^{* *}, m \cdot E=m$. Now, since any $f$ in $A^{*}$ is of the form $f=g \cdot a$ for some $g$ in $A^{*}$ and $a$ in $A$ and since $a \cdot E=a, \quad\langle f, E \cdot m\rangle=$ $\langle g \cdot a, E \cdot m\rangle=\langle g, a \cdot m\rangle=\langle f, m\rangle$ so that $E \cdot m=m$, as well. Thus $A^{* *}$ is unital. Conversely, assume $A^{* *}$ has a unit element $E$. Then $A$ has a BAI $\left(e_{\alpha}\right)_{\alpha \in I}$ that converges to $E$ in the weak* topology of $A^{* *}$. Hence, for any $f$ in $A^{*}$ and $m$ in $A^{* *}$, by the properties of the first Arens multiplication, $\left\langle f \cdot e_{\alpha}, m\right\rangle=\left\langle f, e_{\alpha} \cdot m\right\rangle \rightarrow$ $\langle f, E \cdot m\rangle=\langle f, m\rangle$ so that $f \cdot e_{\alpha} \rightarrow f$ weakly, and by the preceding lemma, $A^{*}$ factors on the left.

The proof of assertion b) is similar to that of a). To prove c), assume that $A^{*}$ factors. Then both algebras $A^{* *}$ and $\left(A^{* *}, \triangle\right)$ are unital. Let $E_{1}$ (resp. $E_{2}$ ) be the unit element of $A^{* *}\left(\operatorname{resp} .\left(A^{* *}, \triangle\right)\right)$. Then, both $E_{1}$ and $E_{2}$ are mixed units. Since $A^{* *}$ is unital, it can have only one mixed unit so that $E_{1}=E_{2}$.

If $A^{*}$ factors on the left (or right) and $B$ is a subalgebra of $A$ with a BAI then $B^{*}$ does not factor in general on the left (or right). Indeed, let $A$ be the group algebra of the discrete group $Z$. Then $A$ being unital, $A^{*}$ factors. Every maximal ideal $B$ of $A$ has a BAI, as is well-known. Since $A$ is separable and WSC, if $B^{*}$ factored, $B$ would be unital, which is not the case. Concerning the quotient algebra we have the following corollary.

Corollary 2.3. Let $A$ be a Banach algebra with a BAI and $I$ a closed ideal of $A$. If $A^{*}$ factors on the left (or right) then so does the dual of the algebra $A / I$.

Proof. Assume that $A^{*}$ factors on the left, and let $\bar{I}^{*}$ be the weak* closure of $I$ in $A^{* *}$. Then $\bar{I}^{*}$ is a closed ideal of $A^{* *}$ and the algebras $(A / I)^{* *}$ and $A^{* *} / \bar{I}^{*}$ are isometrically isomorphic. Since the algebra $A^{* *}$ is unital by the preceding proposition, the quotient algebra $A^{* *} / \bar{I}^{*}$ is unital. Hence the algebra $(A / I)^{* *}$ is unital so that the space $(A / I)^{*}$ factors on the left. The proof of the right version is similar.

Let $A$ be a Banach algebra with a BAI. If $A$ is Arens regular then $A^{*}$ factors, see [27, Theorem 3.1]. In the next example we show that even if $A$ is non unital and non Arens regular then $A^{*}$ may factor.

Example 2.4. Let $B$ be a unital non Arens regular Banach algebra, and let 1 be its unital element. Such a Banach algebra exists of course, take e.g. the group algebra of an infinite discrete group. Let, for each $n$ in $\mathbb{N}, B_{n}$ be a copy of $B$ and $A=\left(\sum_{n=0}^{\infty} \oplus B_{n}\right)_{0}$ be their $c_{0}$-sum. Then $A$ is non unital and non Arens regular. The sequence $\left(e_{n}\right)_{n \in \mathbb{N}}$, where $e_{n}=(1,1, \ldots, 1,0, \ldots, 0, \ldots) \quad(1$ occurs $n$ times), is a BAI for $A$. Since $A^{*}=\left(\sum_{n=1}^{\infty} \oplus B_{n}^{*}\right)_{1}$, the $\ell^{1}$-sum of $B_{n}^{*}$ 's, and since, for each $f=\left(f_{n}\right)$ in $A^{*}, \sum_{n=1}^{\infty}\left\|f_{n}\right\|<\infty$ we have: $e_{n} \cdot f=f \cdot e_{n}=\left(f_{1}, \ldots, f_{n}, 0,0, \ldots\right)$ and $\left\|e_{n} \cdot f-f\right\|=\left\|f \cdot e_{n}-f\right\|=\sum_{p=n+1}^{\infty}\left\|f_{p}\right\| \rightarrow 0$, as $n \rightarrow \infty$. Hence, by Lemma 2.1 above, $A^{*}$ factors. 
In the example below we present a Banach algebra with a BAI whose dual factors on the one side but not on the other side.

Example 2.5. Let $A=K\left(c_{0}\right)$, the operator algebra of all compact linear operators on the sequence space $c_{0}$. Since the space $\ell^{1}=c_{0}^{*}$ has the bounded approximation property, the algebra $A$ has a BAI [4, II.3.10 and II.3.11]. Since $A^{*}=\ell^{1}$ also has the $\operatorname{RNP}$ (= Radon Nikodym property), $A=\ell^{1} \stackrel{\vee}{\oplus} c_{0}$ and $A^{*}=\ell^{\infty} \widehat{\oplus} \ell^{1}$ ([15; p. 122, Theorem 8]) so that $A^{* *}=L\left(\ell^{\infty}\right)$, the operator algebra of all bounded linear operators on $\ell^{\infty}$. Let $i: c_{0} \rightarrow \ell^{\infty}$ and $j: \ell^{1} \rightarrow \ell^{1 * *}$ be the natural injections. Then, as one can show very easily (see [13; p. 49]), for $u$ and $v$ in $L\left(\ell^{\infty}\right)$, the first and second Arens products of $u$ and $v$ are given by:

i) $u \cdot v=u \circ v$, the composition of $u$ and $v$, i.e. the natural multiplication on $L\left(\ell^{\infty}\right)$.

ii) $u \Delta v=\left(j^{*} \circ u^{* *} \circ i^{* *}\right) \circ v$.

Since the algebra $L\left(\ell^{\infty}\right)$ is unital, $A^{*}$ factors on the left. However the algebra $\left(L\left(\ell^{\infty}\right), \triangle\right)$ is not unital, and the space $A^{*}$ does not factor on the right. If in $A$ we define the multiplication by $u \cdot v=v \circ u$ instead of $u \cdot v=u \circ v$ then in this case $A^{*}$ factors on the right but not on the left.

Now let $G$ be a locally compact group and $A=L^{1}(G)$ be its group algebra. It is easy to see that $A^{*}$ factors on the left if and only if it factors on the right if and only if $A$ is unital i.e. $G$ is discrete. The next result shows that whenever $A$ is weakly sequentially complete and has a sequential BAI, the space $A^{*}$ cannot factor on the left or right unless $A$ is unital.

Theorem 2.6. Let $A$ be a weakly sequential complete Banach algebra with a sequential $\mathrm{BAI}\left(e_{n}\right)_{n \in \mathbb{N}}$. Then the following assertiona are equivalent.

a) $A^{*}$ factors on the left.

b) $A^{*}$ factors on the right.

c) A is unital.

Proof. Assume a) holds. Then any $f$ in $A^{*}$ is of the form $f=g \cdot a$ for some $g$ in $A^{*}$ and $a$ in $A$. Since $a e_{n} \rightarrow a,\left\langle f, e_{n}\right\rangle=\left\langle g, a e_{n}\right\rangle \rightarrow\langle g, a\rangle$. This shows that the sequence $\left(e_{n}\right)_{n \in \mathbb{N}}$ is weakly Cauchy. Since $A$ is weakly sequentially complete, it converges weakly to some element $e$ of $A$. It is immediate to see that $e$ is the unit element of $A$. Hence $A$ is unital and $A^{*}$ factors on the right. The rest of the proof is now clear.

We do not know if in this theorem we can drop the word "sequential." Next we present an important corollary of this theorem. The proof of this corollary is immediate since the dual of an Arens regular Banach algebra with a BAI factors [27; Theorem 3.1].

Corollary 2.7. A weakly sequentially complete Banach algebra A with a sequential BAI cannot be Arens regular unless it is unital.

A Banach algebra $A$ is said to be weakly completely continuous (= wcc) if, for each $a$ in $A$, the multiplication operator $x \mapsto a x$ is weakly compact. As another corollary of Theorem 2.6 we have the following result.

Corollary 2.8. Let $A$ be a weakly sequentially complete Banach algebra with a $\operatorname{BAI}\left(e_{\alpha}\right)_{\alpha \in I}$. Then we have: 
a) If $A$ is Arens regular then every separable subalgebra $B_{0}$ of $A$ is contained in a unital separable closed subalgebra $B$ of $A$.

b) If $A$ is Arens regular and wcc then $A$ is reflexive.

Proof. a) Assume that $A$ is Arens regular, and let $B_{0}$ be a separable subalgebra of $A$. Then, by Lemma 3.4 of [27], $B_{0}$ is contained in a separable closed subalgebra $B$ of $A$ that has a (sequential) BAI. Hence $B$, being Arens regular and weakly sequentially complete, is unital by Corollary 2.7 above.

b) Assume that $A$ is Arens regular and wcc. Let $\left(x_{n}\right)_{n \in \mathbb{N}}$ be a bounded sequence in $A$. Then, by the preceding assertion, $\left(x_{n}\right)_{n \in \mathbb{N}}$ is contained in a unital subalgebra $B$. Since $B$ is also wcc and unital, it is reflexive. Hence $\left(x_{n}\right)_{n \in \mathbb{N}}$ has a weakly convergent subsequence, and $A$ is reflexive.

Next we give another class of Banach algebras $A$ for the dual of which left and right factorizations are equivalent.

Proposition 2.9. Let $A$ be a Banach algebra with a sequential BAI $\left(e_{n}\right)_{n \in \mathbb{N}}$ such that $A^{*}$ is weakly sequentially complete. Then $A^{*}$ factors on the left if and only if it factors on the right.

Proof. Assume that $A^{*}$ factors on the left, and let $f$ be an element of $A^{*}$. Then $f$ is of the form $f=g \cdot a$ for some $g$ in $A^{*}$ and $a$ in $A$. Then $\left\langle f, e_{n}\right\rangle=\left\langle g, a e_{n}\right\rangle \rightarrow\langle g, a\rangle$ so that the sequence $\left(e_{n}\right)_{n \in \mathbb{N}}$ is weakly Cauchy. The operator $\Phi_{f}: A \rightarrow A^{*}$, defined by $\Phi_{f}(a)=a \Delta f$, being linear and continuous, the sequence $\left(e_{n} \Delta f\right)_{n \in \mathbb{N}}$ is weakly Cauchy in $A^{*}$. Hence, $A^{*}$ being weakly sequentially complete and $\left(e_{n}\right)_{n \in \mathbb{N}}$ a BAI in $A, e_{n} \triangle f \rightarrow f$ weakly. Hence, by Lemma $2.1, A^{*}$ factors on the right. The proof of the reverse implication is very similar.

The next series of results relates the factorization problem for $A^{*}$ to the topological center problem for $A^{* *}$. Before proceeding we remark that

$$
Z_{1}=\left\{m \in A^{* *}: m \cdot n=m \Delta n \forall n \in A^{* *}\right\}
$$

and

$$
Z_{2}=\left\{m \in A^{* *}: n \cdot m=n \triangle m \forall n \in A^{* *}\right\} .
$$

Proposition 2.10. Let $A$ be a Banach algebra with a BAI. If $A^{*}$ factors on the one side but not on the other side then $Z_{1} \neq Z_{2}$.

Proof. Assume, to be fixed, that $A^{*}$ factors on the left but not on the right. Then, by Proposition 2.2, the algebra $A^{* *}$ is unital. Let $E$ be the unit element of $A^{* *}$. Then $E$ is also a mixed unit so that, for all $n$ in $A^{* *}, \quad n=E \cdot n=E \triangle n$. Hence, by the above remark, $E$ is in $Z_{1}$. Should we have $Z_{1}=Z_{2}, E$ would be in $Z_{2}$ and the algebra $\left(A^{* *}, \triangle\right)$ would be unital, which is not the case by Proposition 2.2. Hence $Z_{1} \neq Z_{2}$.

Now we introduce the following sets, which we shall use in the next sections too.

$$
M_{1}=\left\{m \in A^{* *}: A \cdot m \subseteq A\right\}
$$

and

$$
M_{2}=\left\{m \in A^{* *}: m \cdot A \subseteq A\right\} .
$$


Proposition 2.11. Let $A$ be a Banach algebra with a BAI. Then

a) $A^{*}$ factors on the left if and only if $M_{1} \subseteq Z_{1}$; and

b) $A^{*}$ factors on the right if and only if $M_{2} \subseteq Z_{2}$.

Proof. We shall give the proof of assertion a), that of b) is very similar. Assume first that $A^{*}=A^{*} A$, and let $m$ be an arbitrary element of $M_{1}$. Since, for all $a$ in $A, a m$ is in $A$ and any $f$ in $A^{*}$ is of the form $f=g \cdot a$ for some $g$ in $A^{*}$ and $a$ in $A$, for any $n$ in $A^{* *}$, we have

$$
\begin{aligned}
\langle f, m \Delta n\rangle & =\langle g, a \cdot(m \Delta n)\rangle=\langle g, a \Delta(m \Delta n)\rangle \\
& =\langle g,(a m) \Delta n\rangle=\langle g, a \cdot m \cdot n\rangle=\langle f, m \cdot n\rangle
\end{aligned}
$$

so that $m \triangle n=m \cdot n$. This being true for all $n$ in $A^{* *}, m$ is in $Z_{1}$ so that $M_{1} \subseteq Z_{1}$. Conversely, assume that $M_{1} \subseteq Z_{1}$. Let $E$ be a mixed unit in $A^{* *}$. As, for all $a$ in $A, a \cdot E=a, E$ is in $M_{1}$, so in $Z_{1}$. Hence, for all $n$ in $A^{* *}, E \cdot n=E \Delta n=n$. This shows that $A^{* *}$ is unital so that $A^{*}$ factors on the left.

If the algebra $A$ is commutative then, as one can see immediately, $Z_{1}=Z_{2}$ and $Z_{1}$ is the algebraic center of $A^{* *}$. A similar result holds if the algebra $A$ has a continuous involution. Indeed let $A$ be any Banach algebra and $T: A \rightarrow A$ be a continuous involution. For $a$ in $A$ and $m$ in $A^{* *}$, put $a^{*}=T(a)$ and $m^{*}=T^{* *}(m)$. If a net $\left(a_{\alpha}\right)_{\alpha \in I}$ in $A$ converges to $m$ in the weak* topology of $A^{* *}$ then $a_{\alpha}^{*} \rightarrow m^{*}$ for the same topology. From this it follows that $m^{* *}=T^{* *}\left(m^{*}\right)=m$ and, for any $m, n$ in $A^{* *},(m \cdot n)^{*}=n^{*} \Delta m^{*}$. In view of this fact the next proposition is immediate.

Proposition 2.12. Let $A$ be a Banach algebra with a BAI and a continuous involution $T: A \rightarrow A$. For $m$ in $A^{* *}$, let $m^{*}=T^{* *}(m)$. Then the mapping $h: Z_{1} \rightarrow Z_{2}$ defined by $h(m)=m^{*}$ is an anti-isomorphism i.e. $h(m \cdot n)=h(n) \Delta h(m)$. In particular, $A Z_{1} \subseteq A$ if and only if $Z_{2} A \subseteq A$.

\section{The Topological Center Problem and Weak Sequential Completeness}

Let $A$ be a Banach algebra with a BAI. In this section we first show that if $\widetilde{Z}_{1}$ is the topological center of the algebra $\left(A^{*} A\right)^{*}$ then $A Z_{1}=A \widetilde{Z}_{1}$. This is a very useful result. We then determine $Z_{1}$ in the case where $A$ or $A^{*}$ is weakly sequentially complete and the inclusion $A Z_{1} \subseteq A$ holds. In the last part of this section we study the problem when the equality wap $(A)=A^{*} A$ holds.

Let $A$ be a Banach algebra with a BAI. By $\mathcal{E}$ we denote the set of the mixed units in $A^{* *}$. For each $E$ in $\mathcal{E}$, the mapping $P_{E}: A^{* *} \rightarrow A^{* *}$ defined by $P_{E}(m)=E \cdot m$ is a norm continuous projection that induces the topological direct sum

$$
A^{* *}=P_{E}\left(A^{* *}\right) \oplus\left(I-P_{E}\right)\left(A^{* *}\right),
$$

which we denote simply as

$$
A^{* *}=E \cdot A^{* *} \oplus(1-E) \cdot A^{* *} .
$$

It is easy to see that $(1-E) \cdot A^{* *}$ is a closed two-sided ideal in $A^{* *}$ and that the annihilator $\left(A^{*} A\right)^{\perp}$ of $A^{*} A$ in $A^{* *}$ can be identified with $(1-E) \cdot A^{* *}$. It follows 
that the dual $\left(A^{*} A\right)^{*}$ of $A^{*} A$ can be identified with the closed right ideal $E \cdot A^{* *}$ of $A^{* *}$. This identification induces on $\left(A^{*} A\right)^{*}$ a multiplication for which it is a Banach algebra and the mapping $\theta_{E}: E \cdot A^{* *} \rightarrow\left(A^{*} A\right)^{*}$ defined by

$$
\left\langle\theta_{E}(E \cdot m), f \cdot a\right\rangle=\langle m, f \cdot a\rangle
$$

is a Banach algebra isomorphism, which is an isometry if $\|E\|=1$. The algebra $A$ is a subalgebra of both $A^{* *}$ and $\left(A^{*} A\right)^{*}$. It is important to remark that, for $a$ in $A$ and $\mu$ in $\left(A^{*} A\right)^{*}$, the product $a \mu$ makes sense both as an element of $\left(A^{*} A\right)^{*}$ and as an element of $A^{* *}$. Moreover if $\widetilde{\mu}$ is any Hahn-Banach extension of $\mu$ to $A^{*}$ then $a \cdot \mu=a \cdot \widetilde{\mu}$. Now for $m$ in $A^{* *}$ and $f$ in $A^{*}$ we define the functional

$$
\widehat{f \cdot m}: A^{* *} \rightarrow \mathbb{C}
$$

by $\langle\widehat{f \cdot m}, n\rangle=\langle f, m \cdot n\rangle$. The functional $\widehat{f \cdot m}$ is in $A^{* * *}=A^{*} \oplus A^{\perp}$ but need not be in $A^{*}$. Similarly, for $\mu$ in $\left(A^{*} A\right)^{*}$ and $f$ in $A^{*} A$ we define the functional

$$
\widetilde{f \cdot \mu}:\left(A^{*} A\right)^{*} \rightarrow \mathbb{C}
$$

by $\langle\widetilde{f \cdot \mu}, \lambda\rangle=\langle f, \mu \cdot \lambda\rangle$. The functional $\widetilde{f \cdot \mu}$ is in $\left(A^{*} A\right)^{* *}$ but need not be in $A^{*} A$. The topological center of the algebra $\left(A^{*} A\right)^{*}$ is defined to be the following set

$$
\begin{aligned}
\widetilde{Z}_{1}= & \left\{\mu \in\left(A^{*} A\right)^{*}: \text { the mapping } \lambda \mapsto \mu \cdot \lambda\right. \\
& \left.{\text { is } \text { weak }^{*} \text {-weak }}^{*} \text { continuouns on }\left(A^{*} A\right)^{*}\right\} .
\end{aligned}
$$

The following lemma plays a key role in this paper.

Lemma 3.1. Let $A$ be a Banach algebra with a BAI, $m$ be an element in $A^{* *}$ and $\mu$ be an element in $\left(A^{*} A\right)^{*}$. Then the following assertions hold.

a) $m$ is in $Z_{1}$ if and only if, for each $f$ in $A^{*}$, the functional $\widehat{f \cdot m}$ is in $A^{*}$. If this happens, $\widehat{f} \cdot m=f \Delta m$ and $f \Delta m$ is in $A^{*} A$.

b) $\mu$ is in $\widetilde{Z}_{1}$ if and only if, for each $g$ in $A^{*} A$, the functional $\widetilde{g \cdot \mu}$ is in $A^{*} A$.

c) $\mu$ is in $\widetilde{Z}_{1}$ if and only if, for each a in $A$, a $\mu$ is in $Z_{1}$.

Proof. a) Assume $m$ is in $Z_{1}$, and let $f$ be an element of $A^{*}$. Then, for all $n$ in $A^{* *}$,

$$
\langle\widehat{f \cdot m}, n\rangle=\langle f, m \cdot n\rangle=\langle f, m \Delta n\rangle=\langle f \Delta m, n\rangle
$$

so that $\widehat{f \cdot m}=f \Delta m$, and $\widehat{f \cdot m}$ is in $A^{*}$. Conversely, assume that, for each $f$ in $A^{*}, \widehat{f \cdot m}$ is in $A^{*}$, and let $\left(n_{\alpha}\right)_{\alpha \in I}$ be a weak ${ }^{*}$ convergent net in $A^{* *}$ converging to some $n$. Then

$$
\left\langle f, m \cdot n_{\alpha}\right\rangle=\left\langle\widehat{f \cdot m}, n_{\alpha}\right\rangle \rightarrow\langle\widehat{f \cdot m}, n\rangle=\langle f, m \cdot n\rangle
$$

so that $m$ is in $Z_{1}$. Now assume $m$ is in $Z_{1}$ and $f$ is in $A^{*}$. Let $\left(a_{\alpha}\right)_{\alpha \in I}$ be a net in $A$ that converges weak ${ }^{*}$ in $A^{* *}$ to $m$. Then, since $\widehat{f \cdot m}$ is in $A^{*}$ and for each $n$ in $A^{* *}$,

$$
\left\langle f \cdot a_{\alpha}, n\right\rangle=\left\langle f, a_{\alpha} \cdot n\right\rangle \rightarrow\langle f, m \cdot n\rangle=\langle\widehat{f \cdot m}, n\rangle
$$


we see that $f \cdot a_{\alpha} \rightarrow \widehat{f \cdot m}$ weakly in $A^{*}$. As $A^{*} A$ is a closed subspace of $A^{*}, \widehat{f \cdot m}$ is in $A^{*} A$.

b) Assume first that $\mu$ is in $\widetilde{Z}_{1}$, and let $g$ be in $A^{*} A$. Let $\left(\lambda_{\alpha}\right)_{\alpha \in I}$ be a weak* convergent net in $\left(A^{*} A\right)^{*}$ converging to some $\lambda$. Then, by definition of $\widetilde{Z}_{1}$,

$$
\left\langle\widetilde{g \cdot \mu}, \lambda_{\alpha}\right\rangle=\left\langle g, \mu \cdot \lambda_{\alpha}\right\rangle \rightarrow\langle g, \mu \cdot \lambda\rangle=\langle\widetilde{g \cdot \mu}, \lambda\rangle .
$$

This shows that the functional $\widetilde{g \cdot \mu}$ is in the dual of the space $\left(\left(A^{*} A\right)^{*}\right.$, weak $\left.{ }^{*}\right)$, that is in $A^{*} A$. The converse implication follows from (1) and the definition of $\widetilde{Z}_{1}$.

c) To prove assertion c), let $\mu$ be in $\widetilde{Z}_{1}$. Then, for each $g=f \cdot a$ in $A^{*} A, \widetilde{g \cdot \mu}$ is in $A^{*} A$. Let $n$ be an element of $A^{* *}$ and $\widetilde{n}$ be its restriction to $\left(A^{*} A\right)$. Then $a \mu \cdot \widetilde{n}=a \mu \cdot n$ and

$$
\langle\widetilde{g \cdot \mu}, n\rangle=\langle\widetilde{g \cdot \mu}, \widetilde{n}\rangle=\langle f, a \mu \cdot \widetilde{n}\rangle=\langle f, a \mu \cdot n\rangle=\langle\widehat{f \cdot a \mu}, n\rangle
$$

so that $\widehat{f \cdot a \mu}=\widetilde{g \cdot \mu}$, and $a \mu$ is in $Z_{1}$. From this the converse implication also follows.

Corollary 3.2. For any Banach algebra with a BAI the equality $A Z_{1}=A \widetilde{Z}_{1}$ holds.

Proof. Since $A$ has a BAI, $A \cdot A=A$. By part c) of the preceding lemma, $A \widetilde{Z}_{1} \subseteq Z_{1}$. Hence $A \widetilde{Z}_{1}=A \cdot A \widetilde{Z}_{1} \subseteq A Z_{1}$. To prove the reverse inclusion, we remark that if $m$ is in $Z_{1}$ and $\mu$ is its restriction to $A^{*} A$ then $\mu$ is in $\widetilde{Z}_{1}$ and that, for $a$ in $A, a \mu=a m$. Hence $A Z_{1}=A \widetilde{Z}_{1}$.

As an application of the preceding corollary, let $G$ be any locally compact group and $A=L^{1}(G)$. Then, as proved in [20], for this algebra, $\widetilde{Z}_{1}=M(G)$, the measure algebra of $G$. Since $L^{1}(G)$ is an ideal in $M(G)$, from the above corollary we get the information that $A Z_{1} \subseteq A$. As we shall see below, this kind of inclusion plays a basic role in the determination of $Z_{1}$.

To proceed we need a piece of terminology. A functional $f$ in $A^{*}$ is said to be weakly completely continuous (=wcc) if the linear operator $\Phi_{f}: A \rightarrow A^{*}$ defined by $\Phi_{f}(a)=f \cdot a$ maps weakly Cauchy sequences into weakly convergent ones. (This should not be confused with the definition of wcc Banach algebras used in Section 2.) For instance, if $A$ or $A^{*}$ is weakly sequentially complete or $A$ is Arens regular then every $f$ in $A^{*}$ is wcc. Another notion we need is that of Baire-1 functional in $A^{* *}$. An element $m$ of $A^{* *}$ is said to be Baire-1 if there exists a sequence $\left(a_{n}\right)_{n \in \mathbb{N}}$ in $A$ that converges to $m$ in the weak* topology of $A^{* *}$. The collection of Baire- 1 elements of $A^{* *}$ is denoted by $B_{1}(A)$. The space $B_{1}(A)$ has been introduced by A. Grothendieck in [16] and it is known to be a closed subspace of $A^{* *}[24]$.

Lemma 3.3. Let $A$ be any Banach algebra. Then $B_{1}(A) \subseteq Z_{1}$ if and only if each $f$ in $A^{*}$ is wcc.

Proof. Assume first that $B_{1}(A) \subseteq Z_{1}$. Let $f$ be a functional in $A^{*}$ and $\left(a_{n}\right)_{n \in \mathbb{N}}$ be a weakly Cauchy sequence in $A$. Then $\left(a_{n}\right)_{n \in \mathbb{N}}$ converges in the weak* topology of $A^{* *}$ to some element $m$ of $B_{1}(A)$. As $B_{1}(A) \subseteq Z_{1}, \quad m$ is in $Z_{1}$ so that $\widehat{f \cdot m}=f \Delta m$ is in $A^{*}$. Clearly $f \cdot a_{n} \rightarrow \widehat{f \cdot m}$ for the topology $\sigma\left(A^{*}, A^{* *}\right)$. As $\widehat{f \cdot m}$ is in $A^{*}$, we see that $f \cdot a_{n}=\Phi_{f}\left(a_{n}\right) \rightarrow f \Delta m$ weakly. Hence $f$ is wcc. Conversely, assume 
that each $f$ in $A^{*}$ is wcc. Let $m$ be in $B_{1}(A)$. Then $m=$ weak $^{*}-\lim a_{n}$ for some sequence $\left(a_{n}\right)_{n \in \mathbb{N}}$ in $A$. The sequence $\left(a_{n}\right)_{n \in \mathbb{N}}$ is necessarily weakly Cauchy and, $f$ being wcc, $\left(f \cdot a_{n}\right)_{n \in \mathbb{N}}$ converges weakly in $A^{*}$. The weak limit of the sequence $\left(f \cdot a_{n}\right)_{n \in \mathbb{N}}$ is necessarily $\widehat{f \cdot m}$. Hence, $\widehat{f \cdot m}$ is in $A^{*}$ and, by Lemma $3.1, m$ is in $Z_{1}$.

Theorem 3.4. Let $A$ be a Banach algebra with a sequential $\mathrm{BAI}\left(e_{n}\right)_{n \in \mathbb{N}}$ and such that $A Z_{1} \subseteq A$. Then

a) If $A$ is weakly sequentially complete then $Z_{1}=A$.

b) If $A^{*}$ is weakly sequentially complete then $Z_{1}=B_{1}(A)$.

c) If $A$ is Arens regular then $A^{* *}=B_{1}(A)$.

Proof. a) Assume that $A$ is weakly sequentially complete. Then $B_{1}(A)=A$ and, for each $m$ in $Z_{1}$ and each mixed unit $E, \quad E \cdot m=m$. Thus, if $m$ is in $Z_{1}$, the only weak ${ }^{*}$ cluster point of the sequence $\left(e_{n} \cdot m\right)_{n \in \mathbb{N}}$ is $m$. It follows that $m=$ weak $^{*}-\lim e_{n} \cdot m$ so that $m$ is in $B_{1}(A)$. Hence $Z_{1}=A$.

b) Assume that $A^{*}$ is weakly sequentially complete. Then each $f$ in $A^{*}$ is wcc so that $B_{1}(A) \subseteq Z_{1}$ by Lemma 3.3 . Let $m$ be in $Z_{1}$. Then the sequence $\left(e_{n} \cdot m\right)_{n \in \mathbb{N}}$ is in $A$ and, as we have seen above, $m=$ weak $^{*}-\lim e_{n} \cdot m$. Hence $m$ is in $B_{1}(A)$, and $B_{1}(A)=Z_{1}$.

c) Assume $A$ is Arens regular. Then $Z_{1}=A^{* *}$ so that for each $m$ in $A^{* *}$ the sequence $\left(e_{n} \cdot m\right)_{n \in \mathbb{N}}$ is in $A$. As above, $m=$ weak $^{*}-\lim e_{n} \cdot m$ so that $A^{* *}=B_{1}(A)$.

We remark that if $A$ is a right ideal in its second dual then the inclusion $A Z_{1} \subseteq A$ holds automatically. We also remark that even if $A$ is not separable it may have a sequential BAI, see Example 2.4 above. As an immediate application of the above theorem we give the following corollary, which is due to F. Ghahramani and P. McClure [8]. We remark that these algebras are separable, ideals in their second dual and have BAI's.

Corollary 3.5. Let $A$ be the Volterra algebra $L^{1}(0,1)$ or the weighted convolution algebra $L^{1}\left(\mathbb{R}^{+}, w\right)$ with a regulated weight $w$. Then, for these algebras, $Z_{1}=A$.

We proceed with an application of the topological center notion. To explain the question we want to consider, let $A$ be a Banach algebra with a BAI. If $A$ is a right ideal in its second dual then wap $(A)=A^{*} A$, see e.g. [27, Theorem 3.1.(b)]. However, in the reverse direction, the equality wap $(A)=A^{*} A$ does not imply that $A$ is a right ideal in its second dual since for any Arens regular Banach algebra $A$ with a BAI, the equalities wap $(A)=A^{*}=A^{*} A$ hold. The next theorem deals with this question.

Theorem 3.6. Let $A$ be a Banach algebra with a BAI. Then the following assertions are equivalent.

a) $\operatorname{wap}(A)=A^{*} A$.

b) $A A^{* *} \subseteq Z_{1}$.

c) $A A^{* *} \subseteq A \widetilde{Z}_{1}$.

d) The multiplication of the algebra $\left(A^{*} A\right)^{*}$ is separately continuous in the weak ${ }^{*}$ topology of this space, i.e. $\widetilde{Z}_{1}=\left(A^{*} A\right)^{*}$.

Proof. a) $\Longrightarrow$ b). Assume that $\operatorname{wap}(A)=A^{*} A$. Let $a$ be in $A$ and $m$ be in $A^{* *}$. 
Then, since for each $f$ in $A^{*}, f \cdot a$ is in wap $(A)$, for each $n$ in $A^{* *}$, we have:

$$
\begin{aligned}
\langle f, a m \cdot n\rangle & =\langle f \cdot a, m \cdot n\rangle=\langle f \cdot a, m \Delta n\rangle \\
& =\langle f, a \cdot(m \Delta n)\rangle=\langle f, a \Delta(m \Delta n)\rangle=\langle f,(a m) \Delta n\rangle .
\end{aligned}
$$

These equalities show that $a m$ is in $Z_{1}$. Hence $A A^{* *} \subseteq Z_{1}$.

b) $\Longrightarrow \mathrm{c})$. Assume that $A A^{* *} \subseteq Z_{1}$. Then, since $A$ has a BAI, $A \cdot A=A$ so that $A A^{* *}=A A A^{* *} \subseteq A Z_{1}$. Hence, by Corollary $3.2, A A^{* *} \subseteq A \widetilde{Z}_{1}$.

c) $\Longrightarrow \mathrm{d})$. Assume that $A A^{* *} \subseteq A \widetilde{Z}_{1}$. Let $\mu$ be an element in $\left(A^{*} A\right)^{*}$ and $\left(\lambda_{\alpha}\right)_{\alpha \in I}$ be a net in this algebra converging weak ${ }^{*}$ to some element $\lambda$ of $\left(A^{*} A\right)^{*}$. We have to show that $\mu \cdot \lambda_{\alpha} \rightarrow \mu \cdot \lambda$ in the weak* topology of this space. To this end, let $f \cdot a$ be an arbitrary element in $A^{*} A$. Then, denoting by $\widetilde{\mu}$ any Hahn-Banach extension of $\mu$ to $A^{*}$, we have:

$$
\left\langle f \cdot a, \mu \cdot \lambda_{\alpha}\right\rangle=\left\langle f, a \mu \cdot \lambda_{\alpha}\right\rangle=\left\langle f, a \widetilde{\mu} \cdot \lambda_{\alpha}\right\rangle
$$

As $a \widetilde{\mu}$ is in $A A^{* *}$ and $A A^{* *} \subseteq A \widetilde{Z}_{1}, a \widetilde{\mu}=b \cdot v$ for some $b$ in $A$ and $v$ in $\widetilde{Z}_{1}$. Hence

$$
\begin{aligned}
\left\langle f \cdot a, \mu \cdot \lambda_{\alpha}\right\rangle & =\left\langle f, b v \cdot \lambda_{\alpha}\right\rangle=\left\langle f \cdot b, v \cdot \lambda_{\alpha}\right\rangle \rightarrow\langle f \cdot b, v \cdot \lambda\rangle \\
& =\langle f, b v \cdot \lambda\rangle=\langle f, a \widetilde{\mu} \cdot \lambda\rangle=\langle f \cdot a, \mu \cdot \lambda\rangle .
\end{aligned}
$$

This proves that $\mu$ is in $\widetilde{Z}_{1}$. Hence $\widetilde{Z}_{1}=\left(A^{*} A\right)^{*}$.

d) $\Longrightarrow$ a). Assume that $\widetilde{Z}_{1}=\left(A^{*} A\right)^{*}$. Let $f \cdot a$ be an element in $A^{*} A$. We have to show that the set

$$
H(f \cdot a)=\{f \cdot a \cdot b: b \in A,\|b\| \leq 1\}
$$

is relatively weakly compact. To this end, let $\left(b_{\alpha}\right)_{\alpha \in I}$ be a net in the unit ball of $A$ that converges to an element $m$ of $A^{* *}$ in the weak ${ }^{*}$ topology of this space. Let $\mu$ be the restriction of $m$ to $A^{*} A$. Then, since $\widetilde{Z}_{1}=\left(A^{*} A\right)^{*}, \quad \mu$ is in $\widetilde{Z}_{1}$. Hence, since $a \mu=a m$ and $A \widetilde{Z}_{1}=A Z_{1} \subseteq Z_{1}$, by Lemma 2.1, $\widehat{f \cdot a m}$ is in $A^{*} A$. It follows that the net $\left(f \cdot a b_{\alpha}\right)_{\alpha \in I}$ converges weakly to $\widehat{f \cdot a m}$. This proves that the set $H(f \cdot a)$ is relatively weakly compact, and $A^{*} A=\operatorname{wap}(A)$.

As an immediate corollary of this theorem we have the following result.

Corollary 3.7. Let $A$ be a Banach algebra with a BAI. Then $A$ is a right ideal in $A^{* *}$ if and only if $A Z_{1} \subseteq A$ and $\operatorname{wap}(A)=A^{*} A$.

Hence if $Z_{1}=A$, the equality wap $(A)=A^{*} A$ holds if and only if $A$ is a right ideal in $A^{* *}$. The following result, which is immediate since for $A=L^{1}(G), \quad Z_{1}=A$ [21], is due to E. Granirer [11, p. 62].

Corollary 3.8. Let $G$ be a locally compact group and $A=L^{1}(G)$. Then wap $(A)=$ $\mathrm{LUC}(G)$ if and only if $G$ is compact. 


\section{Topological Center of $\left(A^{*} A\right)^{*}$ and the Multiplier Algebra of $A$}

Let $A$ be a Banach algebra with a BAI bounded by 1 . The main result of this section is that the algebra $\operatorname{RM}(A)$ of all right multipliers of $A$ is isometrically isomorphic to a subalgebra of the topological center $\widetilde{Z}_{1}$ of $\left(A^{*} A\right)^{*}$.

Let $A$ be a Banach algebra with a BAI. In Section 2 we have introduced the set $M_{1}=\left\{m \in A^{* *}: A m \subseteq A\right\}$. Here we introduce the analogue of this set for the algebra $\left(A^{*} A\right)^{*}$, and define the set

$$
\widetilde{M}_{1}=\left\{\mu \in\left(A^{*} A\right)^{*}: A \mu \subseteq A\right\} .
$$

It is clear that, for $m$ in $M_{1}$, the restriction $\mu$ of $m$ to $A^{*} A$ is in $\widetilde{M}_{1}$ and that $A M_{1}=A \widetilde{M}_{1} \subseteq A$. The following result we record here is immediate, see Lemma 3.1.

Proposition 4.1. Let $A$ be a Banach algebra with a BAI. Then $\widetilde{M}_{1}$ is a closed subalgebra of $\left(A^{*} A\right)^{*}$ and $\widetilde{M}_{1} \subseteq \widetilde{Z}_{1}$.

Now we shall determine the set $\widetilde{M}_{1}$ for certain Banach algebras.

Theorem 4.2. Let $G$ be a locally compact group. Then, for the algebra $A=L^{1}(G)$, $\widetilde{M}_{1}=M(G)$.

Proof. Although the multiplication in the algebra $L^{1}(G)$ is the convolution, for $a, b$ in $L^{1}(G)$, we shall continue to denote by $a b$ their product in $L^{1}(G)$. We embed $M(G)$ into $\operatorname{LUC}(G)^{*}=\left(A^{*} A\right)^{*}$ by integration. That is, for $\mu$ in $M(G)$ and $f$ in LUC $(G)$, we define $\langle\mu, f\rangle$ as $\langle\mu, f\rangle=\int_{G} f d \mu$. To prove the equality $\widetilde{M}_{1}=M(G)$, first observe that, since $L^{1}(G)$ is an ideal in $M(G), M(G)$ is contained in $\widetilde{M}_{1}$. To prove the reverse inclusion, let $\mu$ be an element of $\widetilde{M}_{1}$ and $\widetilde{\mu}$ be any Hahn-Banach extension $\mu$ to $L^{\infty}(G)$. Then, for $a$ in $L^{1}(G), a \mu=a \widetilde{\mu}$ so that $a \widetilde{\mu}$ is in $A$. Let $\left(e_{\alpha}\right)_{\alpha \in I}$ be a BAI in $L^{1}(G)$. If necessary passing to a subnet, we can assume that the net $\left(e_{\alpha}\right)_{\alpha \in I}$ converges in the weak ${ }^{*}$ topology of $L^{\infty}(G)^{*}$ to a mixed unit $E$ and the net $\left(e_{\alpha} \mu\right)_{\alpha \in I}$ converges in the topology $\sigma\left(M(G), C_{0}(G)\right)$ of $M(G)$ to an element $\lambda$ of this space. Hence, for $f$ in $C_{0}(G)$

$$
\langle f, \lambda\rangle=\lim _{\alpha}\left\langle f, e_{\alpha} \mu\right\rangle=\lim _{\alpha}\left\langle f, e_{\alpha} \widetilde{\mu}\right\rangle=\langle f, E \cdot \widetilde{\mu}\rangle .
$$

As any $f$ in $C_{0}(G)$ is a weakly almost periodic functional on $L^{1}(G), \quad\langle f, E \cdot \widetilde{\mu}\rangle=$ $\langle f, \widetilde{\mu}\rangle=\langle f, \mu\rangle$, for any $f$ in $C_{0}(G)$. Hence, for any $f$ in $C_{0}(G),\langle f, \lambda\rangle=\langle f, \mu\rangle$. For $a$ in $C_{00}(G)$ (here we consider $C_{00}(G)$ as a subspace of $L^{1}(G)$ ) and $f$ in $C_{0}(G), \quad f \cdot a=$ $\widetilde{a} * f$ (the convolution of $\widetilde{a}(t)=\frac{1}{\Delta(t)} a\left(t^{-1}\right)$ and $f, \Delta$ being the modular function of $G$ ) so that $f \cdot a$ is in $C_{0}(G)$, and

$$
\langle f \cdot a, \lambda\rangle=\langle f \cdot a, \mu\rangle .
$$

This being true for all $a$ in $C_{00}(G)$ and $f$ in $C_{0}(G)$, and $C_{0}(G)$ being weak* dense in $L^{\infty}(G)$ and both $a \lambda$ and $a \mu$ being in $L^{1}(G)$, we conclude that $a \mu=a \lambda$. This being true for all $a$ in $C_{00}(G)$ and this space being norm dense in $L^{1}(G)$, the equality $a \mu=$ $a \lambda$ holds for all $a$ in $L^{1}(G)$. Hence, for any $f$ in $\operatorname{LUC}(G)=L^{\infty}(G) L^{1}(G), \quad\langle f, \mu\rangle=$ $\langle f, \lambda\rangle$. Therefore $\mu=\lambda, \mu$ is in $M(G)$ and the equality $\widetilde{M}_{1}=M(G)$ holds.

Next we determine the set $\widetilde{M}_{1}$ for the Fourier algebra $A(G)$. 
Theorem 4.3. Let $G$ be a locally compact amenable group. Then, for the algebra $A=A(G), \widetilde{M}_{1}=B(G)$.

Proof. We first remark that, the group $G$ being amenable, the algebra $A(G)$ has a BAI [23]. As in Section 1, let $\operatorname{UCB}(\widehat{G})=A(G) \cdot \operatorname{VN}(G)$. Then $B(G)$ is isometrically isomorphic to a closed subalgebra of the algebra $\operatorname{UCB}(\widehat{G})^{*}$ by the map $T: B(G) \rightarrow$ $\operatorname{UCB}(\widehat{G})^{*}$ defined by $\langle T(a), f \cdot b\rangle=\langle f, a b\rangle([19 ;$ p. 49$])$, here $a$ is in $B(G), \quad b$ is in $A(G)$ and $f$ is in $V N(G)$, the dual of $A(G)$. Since $A(G)$ is an ideal in $B(G)$, the inclusion $B(G) \subseteq \widetilde{M}_{1}$ is clear. To prove the reverse inclusion, let $\mu$ be in $\widetilde{M}_{1}$ and $\widetilde{\mu}$ be any Hahn-Banach extension of it to $V N(G)$. Then, for $a$ in $A(G), \quad a \cdot \mu=a \cdot \widetilde{\mu}$ and $a \mu$ is in $A(G)$ by definition of $\widetilde{M}_{1}$. Let $\left(e_{\alpha}\right)_{\alpha \in I}$ be a BAI in $A(G)$. If necessary passing to a subnet, we can assume that the net $\left(e_{\alpha}\right)_{\alpha \in I}$ converges in the weak* topology of $V N(G)^{*}$ to a mixed unit $E$ and the net $\left(e_{\alpha} \widetilde{\mu}\right)_{\alpha \in I}$ converges in the weak* topology of the space $B(G)=\left(C^{*}(G)\right)^{*}$ to an element $\lambda$ of $B(G)$. Here $C^{*}(G)$ is the group $C^{*}$-algebra of the (amenable) group $G$ [7]. Hence, for $f$ in $C^{*}(G)$,

$$
\langle f, \lambda\rangle=\lim _{\alpha}\left\langle f, e_{\alpha} \widetilde{\mu}\right\rangle=\lim _{\alpha}\left\langle f, e_{\alpha} \widetilde{\mu}\right\rangle=\langle f, E \cdot \widetilde{\mu}\rangle .
$$

Since for any $f \cdot a$ in $V N(G) \cdot A(G), \quad\langle f \cdot a, E \cdot \widetilde{\mu}\rangle=\langle f, a \widetilde{\mu}\rangle=\langle f, a \mu\rangle=\langle f \cdot a, \mu\rangle$, for all $f$ in $C^{*}(G)$ we have

$$
\langle f, \lambda\rangle=\langle f, \mu\rangle .
$$

Now if $a$ is in $A(G)$ and $f$ in $C^{*}(G)$, then $f \cdot a$ is in $C^{*}(G)$ so that

$$
\langle f, a \lambda\rangle=\langle f, a \mu\rangle
$$

for all $a$ in $A(G)$ and $f$ in $C^{*}(G)$. Since $C^{*}(G)$ is weak*-dense in $V N(G)$ and $a \mu, a \lambda$ are in $A(G)$, for all $a$ in $A(G)$ and $f$ in $V N(G)$, we have

$$
\langle f \cdot a, \lambda\rangle=\langle f, a \cdot \lambda\rangle=\langle f, a \cdot \mu\rangle=\langle f \cdot a, \mu\rangle .
$$

Hence $\lambda=\mu, \lambda$ is in $B(G)$ and $\widetilde{M}_{1}=B(G)$.

Let $A$ be a Banach algebra. We recall that a bounded linear operator $T: A \rightarrow A$ is said to be a right multiplier if, for all $a, b$ in $A, T(a b)=a T(b)$. We denote by $\mathrm{RM}(A)$ the set of all right multipliers of $A$. The set $\mathrm{RM}(A)$ is a norm closed subalgebra of the algebra $L(A)$ of bounded linear operators on $A$.

Theorem 4.4. Let $A$ be a Banach algebra with a $\mathrm{BAI}\left(e_{\alpha}\right)_{\alpha \in I}$ bounded by 1 . Then the algebra $\mathrm{RM}(A)$ is isometrically isomorphic to the subalgebra $\widetilde{M}_{1}$ of $\widetilde{Z}_{1}$.

Proof. For each $\mu$ in $\widetilde{M}_{1}$, let $T_{\mu}: A \rightarrow A$ be the linear operator defined by $T_{\mu}(a)=a \cdot \mu$. Then $T_{\mu}$ is a right multiplier on $A$ and $\left\|T_{\mu}\right\| \leq\|\mu\|$. Actually $\left\|T_{\mu}\right\|=\|\mu\|$. Indeed, since $\left\|e_{\alpha}\right\| \leq 1$ for all $\alpha$ in $I$,

$$
\left\|T_{\mu}\right\| \geq \sup _{\alpha}\left\|T_{\mu}\left(e_{\alpha}\right)\right\|=\sup _{\alpha}\left\|e_{\alpha} \mu\right\|=\sup _{\alpha} \sup _{\|f \cdot a\| \leq 1}\left|\left\langle e_{\alpha} \mu, f \cdot a\right\rangle\right| .
$$

As, for $f$ in $A^{*}$ and $a$ in $A$,

$$
\begin{gathered}
\left\|f \cdot a e_{\alpha}-f \cdot a\right\| \leq\|f\|\left\|a e_{\alpha}-a\right\| \rightarrow 0, \\
\sup _{\alpha}\left|\left\langle e_{\alpha} \mu, f \cdot a\right\rangle\right| \geq \lim _{\alpha}\left|\left\langle\mu, f \cdot a e_{\alpha}\right\rangle\right|=|\langle\mu, f \cdot a\rangle| .
\end{gathered}
$$


Hence $\sup _{\alpha} \sup _{\|f \cdot a\| \leq 1}\left|\left\langle e_{\alpha} \mu, f \cdot a\right\rangle\right| \geq\|\mu\|$ so that $\left\|T_{\mu}\right\|=\|\mu\|$. It follows that the $\alpha \quad\|f \cdot a\| \leq 1$

mapping $h: \widetilde{M}_{1} \rightarrow \operatorname{RM}(A)$ defined by $h(\mu)=T_{\mu}$ is an isometry. It is clear that $h$ is a Banach algebra homomorphism. To prove that $h$ is onto, let $T$ be any element in $\operatorname{RM}(A)$. As we consider $A$ as a subalgebra of $\left(A^{*} A\right)^{*}$, the net $\left(T\left(e_{\alpha}\right)\right)_{\alpha \in I}$ is in $\left(A^{*} A\right)^{*}$ and, for each $f \cdot a$ in $A^{*} A$,

$$
\left\langle f \cdot a, T\left(e_{\alpha}\right)\right\rangle=\left\langle f, a \cdot T\left(e_{\alpha}\right)\right\rangle=\left\langle f, T\left(a e_{\alpha}\right)\right\rangle \rightarrow\langle f, T(a)\rangle .
$$

This shows that the net $\left(T\left(e_{\alpha}\right)\right)_{\alpha \in I}$ is weak ${ }^{*}$-Cauchy in $\left(A^{*} A\right)^{*}$. Hence it converges to some element $\mu$ of $\left(A^{*} A\right)^{*}$ in the weak* topology of this space. The above equalities show that

$$
\langle f \cdot a, \mu\rangle=\langle f, T(a)\rangle
$$

for all $f$ in $A^{*}$ and $a$ in $A$. Hence $T(a)=a \cdot \mu$ so that $T=T_{\mu}$. This proves that $h$ is onto.

We remark that if $A Z_{1} \subseteq A$ then, in the preceding theorem, $\widetilde{M}_{1}=\widetilde{Z}_{1} \cong \operatorname{RM}(A)$.

As an immediate corollary of the above three theorems we have the following result. Assertion a) of this corollary is due to J. Wendel [28] and assertion b) to A. Derighetti [5].

Corollary 4.5. Let $G$ be a locally compact group. Then

a) For the algebra $A=L^{1}(G)$, the algebra $\mathrm{RM}(A)$ is isometrically isomorphic to $M(G)$.

b) If $G$ is amenable, for $A=A(G)$, the multiplier algebra of $A$ is isometrically isomorphic to $B(G)$.

\section{Distinguishing the Elements of $A$ Among Those of $A^{* *}$}

Throughout this section by $A$ we will denote a Banach algebra with a BAI. As mentioned in the introduction, for certain Banach algebras $A$, including the group algebra $L^{1}(G)$ and, for certain groups $G$, the Fourier algebra $A(G), Z_{1}=A$. Therefore for such algebras $A$, determining the topological center of $A^{* *}$ comes to deciding which elements of $A^{* *}$ are in $A$. In this section we shall present some sufficient, and necessary and sufficient conditions for an element $m$ of $A^{* *}$ to be in $A$ or $Z_{1}$. In particular, we shall give a completely different proof of the fact that for the group algebra $A=L^{1}(G), \quad Z_{1}=A$.

We start with a general result. Before stating this theorem let us remark that, for $f$ in $A^{*}$ and $m$ in $A^{* *}$, the functional $f \Delta m$ is in $A^{*}$ but need not be in $A^{*} A$.

Theorem 5.1. For $m$ in $A^{* *}$ the following two assertions are equivalent.

a) $m$ is in $Z_{1}$.

b) i) $m A \subseteq Z_{1}$, ii) For each $E$ in $\mathcal{E}, \quad m \triangle E=m$; and iii) for each $f$ in $A^{*}, f \triangle m$ is in $A^{*} A$.

Proof. a) $\Longrightarrow$ b). Assume $m$ is in $Z_{1}$. Then since $Z_{1}$ is a subalgebra of $A^{* *}$ and $A \subseteq Z_{1}$, condition i) holds. Condition ii) holds obviously since $m \triangle E=m \cdot E=m$. Condition iii) holds by Lemma 3.1 (a).

b) $\Longrightarrow$ a). Assume b) holds. Let $\left(e_{\alpha}\right)_{\alpha \in I}$ be a BAI in $A$ converging in the weak* topology of $A^{* *}$ to some $E$, and let $n$ be an arbitrary element in $A^{* *}$. By condition iii) and the proof of Lemma 2.1,

$$
\left\langle(f \Delta m) \cdot e_{\alpha}, n\right\rangle \rightarrow\langle f \Delta m, n\rangle=\langle f, m \Delta n\rangle .
$$


On the other hand, since each $m e_{\alpha}$ is in $Z_{1}$ and $m \Delta E=m$ (by (i) and (ii)),

$$
\begin{aligned}
\left\langle(f \Delta m) e_{\alpha}, n\right\rangle & =\left\langle f, m \Delta\left(e_{\alpha} n\right)\right\rangle=\left\langle f, m \Delta e_{\alpha} \Delta n\right\rangle \\
& =\left\langle f,\left(m \cdot e_{\alpha}\right) \Delta n\right\rangle=\left\langle f, m \cdot e_{\alpha} \cdot n\right\rangle \rightarrow\langle f,(m \Delta E) \cdot n\rangle \\
& =\langle f, m \cdot n\rangle
\end{aligned}
$$

so that $m \Delta n=m \cdot n$. This being true for any $n$ in $A^{* *}$, we conclude that $m$ is in $Z_{1}$.

Remarks $5.2 .1^{\circ}$ ) If $Z_{1}=Z_{2}$, assertion b) of the preceding theorem is equivalent to assertion

$\left.\mathrm{b}^{\prime}\right)$ i) $A m \subseteq Z_{1}$; ii) for all $E$ in $\mathcal{E}, E \cdot m=m$; and iii) for all $f$ in $A^{*}, m \cdot f$ is in $A A^{*}$.

$2^{\circ}$ ) If $A$ is an ideal in $A^{* *}$ or $A Z_{1}=A \widetilde{Z}_{1} \subseteq A$ then the condition $A m \subseteq Z_{1}$ holds obviously.

$3^{\circ}$ ) There is a dissymmetry between conditions in assertion b) and the equality $A Z_{1}=A \widetilde{Z}_{1}$ obtained in Corollary 3.2. This dissymmetry remains unexplained.

For the proof of the next theorem we need the following result. For this and related results we refer the reader to the survey paper of G. Godefroy [10; p. 155158], see especially Theorems V.1 and V.3, and Example (4) on page 161.

Lemma 5.3. Let $X$ be a Banach space such that its dual $X^{*}$ is a von Neumann algebra. Then, for $m$ in $X^{* *}$ the following assertions are equivalent.

a) $m$ is in $X$.

b) For any weakly Cauchy sequence $\left(f_{p}\right)_{p \in \mathbb{N}}$ in $X^{*}$ with $f=\sigma\left(X^{*}, X\right)-\lim f_{p}$ we have $\langle f, m\rangle=\lim _{p \rightarrow \infty}\left\langle f_{p}, m\right\rangle$.

Theorem 5.4. Let $G$ be a locally compact group and $A=L^{1}(G)$. Then, for $m$ in $A^{* *}$, the following assertions are equivalent.

a) $m$ is in $A$.

b) i) $A m \subseteq A$, ii) For each $E$ in $\mathcal{E}, E \cdot m=m$; and iii) for each $f$ in $A^{*}, m \cdot f$ is in $A A^{*}$.

Proof. The implication a) $\Longrightarrow$ b) being obvious, we prove the implication b) $\Longrightarrow$ a). Assume that assertion b) holds. Let $\left(f_{p}\right)_{p \in \mathbb{N}}$ be a weakly Cauchy sequence in $L^{\infty}(G)$ and let $f=$ weak $^{*}-\lim f_{p}$. By condition iii), the sequence $\left(m \cdot f_{p}\right)_{p \in \mathbb{N}}$ is in $A A^{*}=\operatorname{RUC}(G)$. Clearly this sequence is weakly Cauchy in $\operatorname{RUC}(G)$. Since, for each $t$ in $G$, the Dirac measure $\delta_{t}$ is in the dual of $\operatorname{RUC}(G)$, the limit

$$
g(t)=\lim _{p \rightarrow \infty}\left(m \cdot f_{p}\right)(t)
$$

exists and $g$ is a bounded measurable function on $G$. On the other hand, since $A m \subseteq A$, for each $a$ in $L^{1}(G)$,

$$
\left\langle m \cdot f_{p}, a\right\rangle=\left\langle f_{p}, a m\right\rangle \rightarrow\langle f, a m\rangle=\langle m \cdot f, a\rangle
$$

so that

$$
m \cdot f=\text { weak }^{*}-\lim m \cdot f_{p} .
$$


Now, for $a$ in $L^{1}(G)$,

$$
\left\langle m \cdot f_{p}, a\right\rangle=\int_{G}\left(m \cdot f_{p}\right)(t) a(t) d \mu(t)
$$

so that by (1) and the Lebesgue Dominated Convergence Theorem,

$$
\langle m \cdot f, a\rangle=\lim \left\langle m \cdot f_{p}, a\right\rangle=\langle g, a\rangle .
$$

This being true for each $a$ in $L^{1}(G)$,

$$
g=m \cdot f \text { locally almost everywhere on } G \text {. }
$$

The function $m \cdot f$ being in $\operatorname{RUC}(G)$ its value $(m \cdot f)(e)$ at the unit element $e$ of $G$ is unambiguously defined. Now let $\left(e_{\alpha}\right)_{\alpha \in I}$ and $\left(\widetilde{e}_{\beta}\right)_{\beta \in J}$ be two BAI in $L^{1}(G)$. By condition ii),

$$
\lim _{\alpha}\left\langle m \cdot f, e_{\alpha}\right\rangle=\lim _{\beta}\left\langle m \cdot f, \widetilde{e}_{\beta}\right\rangle=\langle f, m\rangle
$$

since $m$ is the unique weak ${ }^{*}$ cluster point of the bounded nets $\left(e_{\alpha} \cdot m\right)_{\alpha \in I}$ and $\left(\widetilde{e}_{\beta} \cdot m\right)_{\beta \in J}$. Hence,

$$
\lim _{\alpha}\left\langle g, e_{\alpha}\right\rangle=\lim _{\beta}\left\langle g, \widetilde{e}_{\beta}\right\rangle=\langle f, m\rangle .
$$

From this, by Lemma 2.3 of [18] (this lemma is proved there for $G$ compact but it is also valid for $G$ locally compact) we conclude that the function $g$ is continuous at $e$. Hence by $(3) g(e)=(m \cdot f)(e)$. Next we want to show that $(m \cdot f)(e)=\langle f, m\rangle$. By condition iii), $m \cdot f=a \cdot h$ for some $a$ in $L^{1}(G)$ and $h$ in $L^{\infty}(G)$. The multiplication in $L^{1}(G)$ being convolution, $(a \cdot h)(e)=\langle h, a\rangle$ so that

$$
\begin{aligned}
(m \cdot f)(e) & =\langle h, a\rangle=\langle h, E \cdot a\rangle=\langle a \cdot h, E\rangle \\
& =\langle m \cdot f, E\rangle=\langle f, E \cdot m\rangle=\langle f, m\rangle .
\end{aligned}
$$

Here $E$ is a mixed unit. Thus $g(e)=(m \cdot f)(e)=\langle f, m\rangle$. By definition of $g, \quad g(e)=$ $\lim _{p \rightarrow \infty}\left(m \cdot f_{p}\right)(e)$. By what precedes $\left(m \cdot f_{p}\right)(e)=\left\langle f_{p}, m\right\rangle$ and $g(e)=\langle f, m\rangle$, so that

$$
\langle f, m\rangle=\lim _{p \rightarrow \infty}\left\langle f_{p}, m\right\rangle .
$$

Hence, by the above lemma, $m$ is in $A$ and the implication b) $\Longrightarrow$ a) holds.

As a corollary of the preceding theorem we have the following result which is proved in [21] by a completely different method.

Corollary 5.5. Let $G$ be any locally compact group and $A=L^{1}(G)$. Then $Z_{1}=$ $Z_{2}=A$.

Proof. Let $m$ be an element of $Z_{1}$. As proved in [20], the topological center $\widetilde{Z}_{1}$ of LUC $(G)^{*}$ is the measure algebra $M(G)$ so that $A \widetilde{Z}_{1} \subseteq A$, as well as $\widetilde{Z}_{1} A \subseteq A$. Let $\mu$ be the restriction of $m$ to $\operatorname{LUC}(G)$. Then $\mu$ is in $M(G)$ so that, for each $a$ in $A, \mu a$ is in $A$. Now, for each $f$ in $A^{*}$ and $x$ in $A$, since $x \mu=x m$, we have:

$$
\langle m a \cdot f, x\rangle=\langle f, x m a\rangle=\langle f, x \mu a\rangle=\langle\mu a \cdot f, x\rangle .
$$


This being true for every $x$ in $A, m a \cdot f=\mu a \cdot f$. It follows $m a \cdot f$ is in $A A^{*}$ and we have:

i) $A m a \subseteq A$, ii) $\forall E \in \mathcal{E}, E \cdot m a=m a$; and $\quad$ iii) $\forall f \in A^{*}, m a \cdot f \in A A^{*}$.

From this, by the preceding theorem, we deduce that $m a$ is in $A$. Now let $T: A \rightarrow$ $A, T(a)=a^{*}$ be the natural involution of $A$. As was remarked in Proposition 2.12, $T^{* *}\left(Z_{1}\right)=Z_{2}$. Put $m^{*}=T^{* *}(m)$. Then $m^{*}$ is in $Z_{2}$ and, since $m A \subseteq A$ by what precedes, $A m^{*} \subseteq A$. Hence, since $m^{*}$ in $Z_{2}$, we have:

i) $A m^{*} \subseteq A$, ii) $\forall E \in \mathcal{E}, E \cdot m^{*}=m^{*}$, and $\quad$ iii) $\forall f \in A^{*}, m^{*} \cdot f \in A A^{*}$. From this, again by the preceding theorem, we conclude that $m^{*}$ is in $A$. Hence $Z_{2}=T^{* *}\left(Z_{1}\right)=A$. Since $Z_{1}=T^{* *}\left(Z_{2}\right)$ and $A=T^{* *}(A)$, we get $Z_{1}=Z_{2}=A$.

In the previous sections we have introduced the sets

$$
\begin{aligned}
& M_{1}=\left\{m \in A^{* *}: A m \subseteq A\right\} \text { and } \\
& \widetilde{M}_{1}=\left\{\mu \in\left(A^{*} A\right)^{*}: A \mu \subseteq A\right\}
\end{aligned}
$$

To avoid long paraphrasing we introduce two more sets below. The first of these sets is the following

$$
\mathcal{F}_{1}=\left\{m \in A^{* *}: E \cdot m=m \text { for all } E \text { in } \mathcal{E}\right\} .
$$

It is immediate to see that $Z_{1} \subseteq \mathcal{F}_{1}$ and $A A^{* *} \subset \mathcal{F}_{1}$ and that

$$
\mathcal{F}_{1}=\bigcap_{E \in \mathcal{E}} E \cdot A^{* *}
$$

From the last equality it is clear that $\mathcal{F}_{1}$ is a closed right ideal in $A^{* *}$. The following immediate characterization of $\mathcal{F}_{1}$ will be useful.

Proposition 5.6. Let $m$ be an element in $A^{* *}$. Then $m$ is in $\mathcal{F}_{1}$ if and only if, for each $\operatorname{BAI}\left(e_{\alpha}\right)_{\alpha \in I}$ in $A, \quad m=$ weak $^{*}-\lim e_{\alpha} \cdot m$.

Now let $\mu$ be an element in $\widetilde{M}_{1}$ and let $\widetilde{\mu}$ be any Hahn-Banach extension of $\mu$ to $A^{*}$. Since $A \mu \subseteq A$, for each BAI $\left(e_{\alpha}\right)_{\alpha \in I}$ in $A$ converging weak* in $A^{* *}$ to an element $E$ of $\mathcal{E}$, the element

$$
E \cdot \widetilde{\mu}=\text { weak }^{*}-\lim e_{\alpha} \widetilde{\mu}=\text { weak }^{*}-\lim e_{\alpha} \mu
$$

of $A^{* *}$ does not depend on the Hahn-Banach extension $\widetilde{\mu}$ of $\mu$ we choose. Hence, for $E$ in $\mathcal{E}$, the mapping

$$
\Gamma_{E}: \widetilde{M}_{1} \rightarrow M_{1}
$$

defined by $\Gamma_{E}(\mu)=E \cdot \widetilde{\mu}$ is well-defined. We put

$$
\Lambda_{1}=\bigcap_{E \in \mathcal{E}} \Gamma_{E}\left(\widetilde{M}_{1}\right)
$$

We present below two characterizations of $\Lambda_{1}$. 
Proposition 5.7. For an element $m$ in $A^{* *}$, the following assertions are equivalent.

a) $m$ is in $\Lambda_{1}$.

b) There is an element $\mu$ in $\widetilde{M}_{1}$ such that, for all $E$ in $\mathcal{E}, m=\Gamma_{E}(\mu)$.

c) $m$ is in $\mathcal{F}_{1} \cap M_{1}$.

Proof. a) $\Longrightarrow$ b). Assume $m$ is in $\Lambda_{1}$, and let $\mu$ be the restriction of $m$ to $A^{*} A$. Then $\mu$ is in $\widetilde{M}_{1}$. On the other hand, since $m$ is in $\Lambda_{1}$, for each $E$ in $\mathcal{E}$, there exists an element $\mu_{E}$ in $\widetilde{M}_{1}$ such that $m=\Gamma_{E}\left(\mu_{E}\right)=E \cdot \widetilde{\mu}_{E}$, where $\widetilde{\mu}_{E}$ is a Hahn-Banach extension of $\mu_{E}$ to $A^{*}$. Hence, for each $a$ in $A, a m=a \mu=a \cdot\left(E \cdot \widetilde{\mu}_{E}\right)=a \mu_{E}$. So, for any $a$ in $A$ and $f$ in $A^{*},\langle f, a \mu\rangle=\left\langle f, a \mu_{E}\right\rangle$. That is, $\langle f \cdot a, \mu\rangle=\left\langle f \cdot a, \mu_{E}\right\rangle$. This shows that $\mu=\mu_{E}$ and $m=\Gamma_{E}(\mu)$ for all $E$ in $\mathcal{E}$.

b) $\Longrightarrow$ c). Assume that, for some $\mu$ in $\widetilde{M}_{1}, m=\Gamma_{E}(\mu)$ for all $E$ in $\mathcal{E}$. Since $m=E \cdot \widetilde{\mu}$ for all $E$ in $\mathcal{E}$ and any Hahn-Banach extension $\widetilde{\mu}$ of $\mu$ to $A^{*}, E \cdot m=m$ for all $E$ in $\mathcal{E}$. Hence $m$ is in $\mathcal{F}_{1} \cap M_{1}$.

c) $\Longrightarrow$ a). Assume $m$ is in $\mathcal{F}_{1} \cap M_{1}$, and let $\mu$ be the restriction of $m$ to $A^{*} A$. Then, since $m$ is a Hahn-Banach extension of $\mu$ to $A^{*}$, for each $E$ in $\mathcal{E}, \Gamma_{E}(\mu)=E \cdot m=m$ so that $m$ is in $\Lambda_{1}$.

It is clear that $Z_{1} \subseteq \Lambda_{1}$ if and only if $A \widetilde{Z}_{1} \subseteq A$. It is also clear that we always have $A \subseteq \Lambda_{1}$. Therefore the equality $Z_{1}=A$ holds if and only if $A \widetilde{Z}_{1} \subseteq A$ and $\Lambda_{1}=A$. Next we want to find out when the equality $\Lambda_{1}=A$ holds. First a piece of terminology.

Definition 5.8. We say that $\mathcal{E}$ distinguishes the points of $\widetilde{M}_{1} \backslash A$ from those of $A$ if, for $\mu$ in $\widetilde{M}_{1} \backslash A$, there exist $E_{1}$ and $E_{2}$ in $\mathcal{E}$ such that $\Gamma_{E_{1}}(\mu) \neq \Gamma_{E_{2}}(\mu)$.

The next result shows once more the role of weak sequential completeness in this kind of question.

Proposition 5.9. If $A$ is weakly sequentially complete and has a sequential BAI $\left(e_{p}\right)_{p \in \mathbb{N}}$ then $\mathcal{E}$ distinguishes the points of $\widetilde{M}_{1} \backslash A$ from those of $A$.

Proof. Let $\mu$ be in $\widetilde{M}_{1}$. Assume that for any two mixed units $E, E_{1}, \Gamma_{E}(\mu)=$ $\Gamma_{E_{1}}(\mu)$. Then the element $m=\Gamma_{E}(\mu)$ is in $\Lambda_{1}$ by Proposition 5.7. Hence, by Proposition 5.6, $m=$ weak $^{*}-\lim e_{p} \cdot m$. This shows that the sequence $\left(e_{p} \cdot m\right)$, which is in $A$, is weakly Cauchy. Hence, since $A$ is weakly sequentially complete, $m$ is in $A$. As $m=E \cdot \widetilde{\mu}$, where $\widetilde{\mu}$ is a Hahn-Banach extension of $\mu$ to $A^{*}$, for any element $f \cdot a$ of $A^{*} A$, we have $\langle f \cdot a, m\rangle=\langle f \cdot a, E \cdot \widetilde{\mu}\rangle=\langle f \cdot a, \mu\rangle$. This proves that, as elements in $\left(A^{*} A\right)^{*}, m=\mu$. Since $m$ is in $A, \mu$ is in $A$. This proves that $\mathcal{E}$ distinguishes the points of $\widetilde{M}_{1} \backslash A$ from those of $A$.

For $\mu$ in $\left(A^{*} A\right)^{*}$, let $H(\mu)$ denote the set of all the Hahn-Banach extensions of $\mu$ in $A^{* *}$. For $m$ in $Z_{1}$, the restriction $\mu$ of $m$ to $A^{*} A$ is in $\widetilde{Z}_{1}$. But for $\mu$ in $\widetilde{Z}_{1}$ we may have $H(\mu) \cap Z_{1}=\phi$.

Lemma 5.10. Following assertions are equivalent.

a) $\Lambda_{1}=A$.

b) $\mathcal{E}$ distinguishes the points of $\widetilde{M}_{1} \backslash A$ from those of $A$.

c) For each $\mu$ in $\widetilde{M}_{1} \backslash A, H(\mu) \cap \mathcal{F}_{1}=\phi$. 
Proof. a) $\Longrightarrow$ b). Let $\mu$ be in $\widetilde{M}_{1}$ and be such that, for any two mixed units $E, E_{1}, \Gamma_{E}(\mu)=\Gamma_{E_{1}}(\mu)$. Then the element $m=\Gamma_{E}(\mu)$, is in $\Lambda_{1}$, so in $A$. As in the proof of the preceding proposition, from this we conclude that $\mu$ is in $A$ so that the implication a) $\Longrightarrow$ b) holds.

b) $\Longrightarrow$ c). Let $\mu$ be in $\widetilde{M}_{1} \backslash A$, and assume that $H(\mu) \cap \mathcal{F}_{1} \neq \phi$. Then, any $\widetilde{\mu}$ in $H(\mu) \cap \mathcal{F}_{1}$ is in $\Lambda_{1}$ so that, for any two mixed units $E, E_{1}, \Gamma_{E}(\mu)=\Gamma_{E_{1}}(\mu)=\mu$. However, by b), this is not possible. Hence the implication b) $\Longrightarrow$ c) holds.

c) $\Longrightarrow$ a). Assume c) holds. As we always have $A \subseteq \Lambda_{1}$, if we had $A \neq \Lambda_{1}$ we would have an $m$ in $\Lambda_{1} \backslash A$. Let $\mu$ be the restriction of this $m$ to $A^{*} A$. Then $\mu$ is in $\widetilde{M}_{1} \backslash A$ and, by c), $H(\mu) \cap \mathcal{F}_{1}=\phi$. However this is not possible since $m$ is in the intersection $H(\mu) \cap \mathcal{F}_{1}$. Thus the implication c) $\Longrightarrow$ a) also holds.

An important consequence of the preceding lemma is the following corollary.

Corollary 5.11. Assume that $\mathcal{E}$ distinguishes the points of $\widetilde{M}_{1} \backslash A$ from those of A. Then $Z_{1}=A$ if and only if $A \widetilde{Z}_{1} \subseteq A$.

Proof. If $A \widetilde{Z}_{1} \subseteq A$ then, since $A Z_{1}=A \widetilde{Z}_{1}, \quad Z_{1} \subseteq \Lambda_{1}$. Hence, by the preceding lemma, $Z_{1}=A$. The converse is obvious.

Thus if the algebra of right multipliers $\operatorname{RM}(\mathrm{A})$ of $A$ coincides with $\widetilde{Z}_{1}$ and $\mathcal{E}$ distinguishes the points of $\widetilde{Z}_{1} \backslash A$ from those of $A$ then $Z_{1}=A$. For instance, if $G$ is any locally compact group and $A=L^{1}(G)$, then, as we have seen in Section 4, $\operatorname{RM}(\mathrm{A})=\widetilde{M}_{1}=\widetilde{Z}_{1}[20]$ and, by Proposition 2.4 of $[9], \mathcal{E}$ distinguishes the points of $\widetilde{Z}_{1} \backslash A$ from those of $A$. Hence we get yet another proof of the fact that the topological center of the algebra $L^{1}(G)^{* *}$ is $L^{1}(G)$.

In the next theorem we have collected some of the results obtained above.

Theorem 5.12. For any Banach algebra $A$ with a BAI the following assertions are equivalent.

a) $Z_{1}=\Lambda_{1}=A$.

b) $A \widetilde{Z}_{1} \subseteq A$ and $\mathcal{E}$ distinguishes the points of $\widetilde{Z}_{1} \backslash A$ from those of $A$.

c) $A \widetilde{Z}_{1} \subseteq A$ and, for each $\mu$ in $\widetilde{Z}_{1} \backslash A, H(\mu) \cap \mathcal{F}=\emptyset$.

We finish this section with an application of this theorem.

Lemma 5.13. Let $G$ be an amenable $\sigma$-compact locally compact group and $A=$ $A(G)$. Then $\mathcal{E}$ distinguishes the points of $\widetilde{M}_{1} \backslash A$ from those of $A$.

Proof. Since the algebra $A(G)$ is weakly sequentially complete, it suffices to show that $A$ has a sequential BAI by the above Proposition 5.9. Since $G$ is amenable, $A(G)$ has a bounded approximate identity $\left(u_{\alpha}\right)_{\alpha \in I}$ consisting of positive definite functions such that $\left\|u_{\alpha}\right\|=u_{\alpha}(e)=1$ and $u_{\alpha} \rightarrow 1$ in the weak* topology of $B(G)$. By $[12 ;$ p. 463$]$ the net $\left(u_{\alpha}\right)_{\alpha \in I}$ converges uniformly on each compact subset of $G$ to the constant function 1 . Since $G$ is $\sigma$-compact, from the net $\left(u_{\alpha}\right)_{\alpha \in I}$ we can extract a subsequence $\left(\phi_{n}\right)_{n \in \mathbb{N}}$ which converges to 1 uniformly on each compact subset of $G$. Again by [12; p. 463], we conclude that for each $u$ in $A(G),\left\|\phi_{n} \cdot u-u\right\| \rightarrow 0$. Hence $A(G)$ has a sequential BAI.

The topological center problem for the algebra $\operatorname{UCB}(\widehat{G})^{*}$ is studied in the paper [22], where it is shown that for a large class of locally compact groups $\widetilde{Z}_{1}=B(G)$. The following result is now obvious. See also [22; Theorem 6.4]. 
Corollary 5.14. Let $G$ be an amenable $\sigma$-compact group, and $A=A(G)$. Then $Z_{1}=A(G)$ whenever $\widetilde{Z}_{1}=B(G)$.

\section{Remarks AND Questions}

Let $A$ be a Banach algebra with a BAI. Concerning the problems tackled in this paper a certain number of questions remain unsolved. Here we have collected some of them in the form of remark or question.

a) We have seen that $A^{*}$ may factor on one side without factoring on the other side. However we do not know anything about the structure of these algebras.

b) If $A^{*}$ is separable does $A^{*}$ necessarily factor on the left or right?

c) Is there a non unital, non Arens regular weakly sequentially complete Banach algebra with a BAI? The answer to this question, in view of the result given in Section 2 , seems to be negative.

d) Does the equality $A^{*} A=A A^{*}$ imply that $Z_{1}=Z_{2}$ ?

e) Does the inclusion $A Z_{1} \subseteq A$ imply that $Z_{2} A \subseteq A$ ?

f) If $A$ is weakly sequentially complete and non unital, does the topological center $\widetilde{Z}_{1}$ of $\left(A^{*} A\right)^{*}$ coincide with the algebra of right multipliers $\mathrm{RM}(\mathrm{A})$ of $A$ ?

g) If $A$ is weakly sequentially complete and non unital, does $\mathcal{E}$ distinguish the points of $\widetilde{M}_{1} \backslash A$ from those of $A$ ?

h) Does Theorem 5.4 extend to the algebra $A(G)$ of a locally compact amenable group $G$ ?

i) Let $A$ be weakly sequentially complete and let $m$ be in $Z_{1}$. Consider the mapping $\tau_{m}: A^{*} \rightarrow A^{*}, \quad \tau_{m}(f)=f \Delta m$ (see Lemma 3.1). Are the sets ker $\tau_{m}$ and $\tau_{m}\left(A_{1}^{*}\right)$, where $A_{1}^{*}$ is the closed unit ball of $A^{*}$, weak ${ }^{*}$ closed? Is this the case if $A^{*}$ is a von Neumann algebra?

j) If $Z_{1}=Z_{2}=A$, must $A$ be weakly sequentially complete? $Z_{2}=A$ alone does not imply that $A$ is weakly sequentially complete. Indeed, if $A=K\left(c_{0}\right)$ as in Example 2.5, then one can easily show that $Z_{1}=\left\{u^{*}: u \in L\left(\ell^{1}\right)\right\}$ and $Z_{2}=K\left(c_{0}\right)$. However $A=K\left(c_{0}\right)=\ell^{1} \stackrel{\vee}{\otimes} c_{0}$ is not weakly sequentially complete since $c_{0}$ is not so.

k) In all the cases known to us, $\widetilde{Z}_{1}$ is the dual of a closed subspace of $A^{*}$ (e.g. $\left.M(G)=C_{0}(G)^{*}\right)$. We do not know if this is always so.

\section{REFERENCES}

[1] Arens, R., The adjoint of a bilinear operation, Proc. Amer. Math. Soc. 2 (1951), 839-848. MR 13:659f

[2] Bonsall, F.F. and Duncan, J., Complete Normed Algebras, Springer-Verlag, Berlin, 1973. MR 54:11013

[3] Cecchini, C. and Zappa, A., Some results on the center of an algebra of operators on $V N(G)$ for the Heisenberg group, Canad. J. Math. 33 (1981), 1469-1486. MR 83f:22003

[4] Cigler, J., Losert, V. and Michor, P., Banach modules and functors on categories of Banach spaces, Lecture Notes in Pure and Appl. Math., 46, Marcel Dekker, 1979. MR 80j:46112

[5] Derighetti, A., Some results on the Fourier-Stieltjes algebra of a locally compact group, Comm. Math. Helv. 45 (1970), 219-228. MR 54:856

[6] Diestel, J. and Uhl, J.J., Jr., Vector measures, Math. Surveys, Vol. 15, Amer. Math. Soc., Providence, RI, 1977. MR 56:12216

[7] Eymard, P., L'algèbre de Fourier d'un groupe localement compact, Bull. Soc. Math. France 92 (1964), 181-236. MR 37:4208 
[8] Ghahramani, F. and McClure, J. P., Module homomorphisms of the dual modules of convolution Banach algebras, Canad. Math. Bull. (2) 35 (1992), 180-185. MR 93f:43004

[9] Ghahramani, F., Lau, A.T., and Losert, V., Isometric isomorphisms between Banach algebra related to locally compact groups, Trans. Amer. Math. Soc. 321 (1990), 273-283. MR 90m: 43010

[10] Godefroy, G., Existence and uniqueness of isomorphic preduals: a survey, Contemporary Math. 85 (1989), 131-193. MR 90b:46035

[11] Granirer, E., Exposed points of convex sets and weak sequential convergence, Mem. Amer. Math. Soc., No. 123 (1972). MR 51:1343

[12] Granirer, E. and Leinert, M., On some topologies which coincide on the unit sphere of the Fourier-Stieltjes algebra $B(G)$ and the measure algebra $M(G)$, Rocky Mountain J. Math. 11 (1981), 459-472. MR 85f:43009

[13] Grosser, M., Arens semi-regular Banach algebras, Monatsh. Math. 98 (1984), 41-52. MR 86d: 46042

[14] Grosser, M. and Losert, V., The norm-strict bidual of a Banach algebra and the dual of $C_{u}(G)$, Manuscripta Math. 45 (1984), 127-46. MR 86b:46073

[15] Grothendieck, A., Produits tensoriels topologiques et espaces nucléaires, Mem. Amer. Math. Soc., No. 16 (1955). MR 17:877d

[16] Grothendieck, A., Critères de compacité dans les espaces functionels généraux, Amer. J. Math. 74 (1975), 168-186. MR 13:857e

[17] Hewitt, E. and Ross, K., Abstract Harmonic Analysis, Vols. I and II, New York, 1970. MR 41:7378

[18] Isik, N., Pym, J.S. and Ülger, A., The second dual of the group algebra of a compact group J. London Math. Soc. (2) 35 (1987), 135-148. MR 88f:43012

[19] Lau, A.T., Uniformly continuous functionals of the Fourier algebra of any locally compact group, Trans. Amer. Math. Soc. 251 (1979), 39-59. MR 80m:43009

[20] Lau, A.T., Continuity of Arens multiplication on the dual space of bounded uniformly continuous functions on locally compact groups and topological semigroups, Math. Proc. Camb. Philos. Soc. 99 (1986), 273-283. MR 87i:43001

[21] Lau, A.T. and Losert, V., On the second conjugate algebra of a locally compact group, J. London Math. Soc. 37 (1988), 464-470. MR 89e:43007

[22] Lau, A.T. and Losert, V., The $C^{*}$-algebra generated by operators with compact support on a locally compact group, Journal of Functional Analysis 112 (1993), 1-30. MR 94d:22005

[23] Leptin, H., Sur l'algèbre de Fourier d'un group localement compact, C.R. Acad. Sci. Paris Sér. A-B 266 (1968), 1180-1182. MR 39:362

[24] McWilliams, R.D., A note on weak sequential convergence, Pacific J. Math. 12 (1962), 333335. MR 26:6724

[25] Parsons, D.J., The center of the second dual of a commutative semigroup algebra, Math. Proc. Camb. Phil. Soc. 95 (1984), 71-92. MR 85c:43004

[26] Pym, J.S., The convolution of functionals on spaces of bounded functions, Proc. London Math. Soc. 15 (1965), 84-104. MR 30:3367

[27] Ülger, A., Arens regularity sometimes implies the RNP, Pacific J. Math. 143 (1990), 377399. MR 92d:46127

[28] Wendel, J.G., Left centralizers and isomorphisms of group algebras, Pacific J. Math. 2 (1952), 251-261. MR 14:262c

[29] Zappa, A., The center of the convolution algebra $C_{u}(G)$, Rend. Sem. Math. Univ. Padova 52 (1974), 71-84. MR 53:14014

Department of Mathematics, University of Alberta, Edmonton, Alberta, Canada T6G 2G1

E-mail address: tlau@vega.math.ualberta.ca

Department of Mathematics, Bō̄azici University, 80815 Bebek-Istanbul, Turkey

E-mail address: ulger@boun.edu.tr 